\title{
THE SPACE OF MINIMAL PRIME IDEALS OF A COMMUTATIVE RING $\left.{ }^{(}\right)$
}

\author{
BY \\ M. HENRIKSEN AND M. JERISON
}

Introduction. Of the various spaces of ideal of rings that have been studied (see [1], for example) we are focusing attention on the space of minimal prime ideals because of its special role in the case of rings of continuous functions.

For the simplest manifestation of this role, consider the compact space $\boldsymbol{N}^{*}$ obtained from the discrete space of positive integers $\boldsymbol{N}$ by adjoining a single point, $\infty$. In the ring $C\left(N^{*}\right)$ of all continuous real-valued functions on $N^{*}$, the maximal ideals are very easy to describe. They are in one-one correspondence with the points of $\boldsymbol{N}^{*}$, and are given by

$$
\boldsymbol{M}_{p}=\left\{f \in C\left(\boldsymbol{N}^{*}\right): f(p)=0\right\}, \quad\left(p \in \boldsymbol{N}^{*}\right) .
$$

If $p \in \boldsymbol{N}$, the maximal ideal $\boldsymbol{M}_{p}$ happens also to be a minimal prime ideal. But $M_{\infty}$ is not a minimal prime ideal. In fact, it contains $2^{c}$ distinct minimal prime ideals - they are in one-one correspondence with the ultrafilters on $N$ that converge to $\infty$. The space of all minimal prime ideals of $C\left(N^{*}\right)$ with the hull-kernel topology (see $\$ 2$ below) is homeomorphic with the space $\beta N$, the Stone-Čech compactification of $N$. The facts about the minimal prime ideals of $C\left(N^{*}\right)$ were first discovered by Kohls [6] and are presented in detail in [2, Chapter 14].

The present paper is devoted to the space of minimal prime ideals of a more-or-less arbitrary commutative ring. Rings $C(X)$ of continuous functions on topological spaces $X$ appear only in $\$ 5$ where they serve largely to provide significant examples. Detailed study of the relation between the space of minimal prime ideals of $C(X)$ and the space $X$ will appear in a later publication.

Some of our results about minimal prime ideals, especially those in $\$ \$ 1$ and 2 , where obtained independently and in a more general setting by J. Kist [5].

A summary of the present paper appears in the "Proceedings of the Symposium on General Topology and its Relation to Modern Analysis and Algebra," Prague, 1961.

1. Minimal prime ideals. Throughout this paper, $A$ will denote a commutative ring. By a minimal prime ideal of $A$, we shall mean a proper prime

Received by the editors December 13, 1963.

$\left({ }^{1}\right)$ This research was supported by the National Science Foundation. 
ideal that contains no smaller prime ideal. Thus, for example, if $A$ is an integral domain, then (0) is the only minimal prime ideal of $A$. The set of all minimal prime ideals of a ring $A$ will be denoted by $\mathscr{P}(A)$, or simply by $\mathscr{P}$. The following facts about prime ideals may be found in [7].

(i) If an ideal $I$ of $A$ is contained in a prime ideal $P$, then there is a prime ideal $P_{0}$ of $A$ that is minimal with respect to the property that $I \subset P_{0} \subset P$.

(ii) The intersection of all prime ideals of $A$, which is also the intersection of all minimal prime ideals of $A$, is the ideal $\eta(A)$ of nilpotent elements in $A$.

(iii) If $I$ is an ideal that is disjoint from a multiplicative system $M$ (i.e., $M$ is closed under multiplication, and $0 \notin M$ ), then $I$ is contained in a prime ideal that is disjoint from $M$.

The following lemma provides the effective criterion for determining when a prime ideal is minimal [5, Lemma 3.1].

1.1. Lemma. A prime ideal $P$ of $A$ is minimal if and only if for each $x \in P$ there exists $a \in A \sim P$ such that ax is nilpotent.

1.2. Corollary. Any member of a minimal prime ideal is a zero-divisor.

Proof. Let $x$ belong to the minimal prime ideal $P$, and let $a \in A \sim P$ and $n \in N$ satisfy $(a x)^{n}=0$. Now, $a^{n} \neq 0$ since $a^{n} \in A \sim P$. Let $k$ be the smallest positive integer such that $a^{n} x^{k}=0$. If $k=1$, we are done. Otherwise, $a^{n} x^{k-1} \neq 0$ and $\left(a^{n} x^{k-1}\right) x=0$.

2. The space of minimal prime ideals. For any subset $S$ of $A$, we define, as usual, the hull of $S$ :

$$
h(S)=\{P \in \mathscr{P}: S \subset P\} .
$$

For any subset $\mathscr{S}$ of $\mathscr{P}(A)$, the kernel of $\mathscr{S}$ is defined as

$$
k(\mathscr{S})=\bigcap\{P: P \in \mathscr{S}\} .
$$

A subset $\mathscr{S}$ of $\mathscr{P}$ is said to be closed if $\mathscr{S}=h k(\mathscr{S})$. With this notion of closed set, $\mathscr{P}(A)$ becomes a topological space, as is well known. The hull of any subset of $A$ is a closed set in $\mathscr{P}(A)$, and the family of all sets of the form $h(a), a \in A$, is a base for the closed sets. From now on, we shall consider $\mathscr{P}(A)$ as a topological space with this topology.

2.1. Theorem. Let $I$ be an ideal of $A$. The mapping $\tau: h(I) \rightarrow \mathscr{P}(A / I)$ defined by

$$
\tau(P)=P / I, \quad P \in h(I),
$$

is a homeomorphism of $h(I)(\subset \mathscr{P}(A))$ onto a subspace of $\mathscr{P}(A / I)$.

The routine proof of this theorem will be omitted.

Generally speaking, $\tau[h(I)]$ is not all of $\mathscr{P}(A / I)$ nor is it dense in $\mathscr{P}(A / I)$. Thus, if $I$ is a maximal, nonminimal, prime ideal in a ring with 
unity-e.g., the ideal (2) in the ring of integers-then $h(I)$ is empty while $\mathscr{P}(A / I)$ consists of one point. In some important special cases, however, we can assert that $\tau[h(I)]=\mathscr{P}(A / I)$, in particular, when $I=\eta(A)$.

2.2. Theorem. $\mathscr{P}(A)$ and $\mathscr{P}(A / \eta(A))$ are homeomorphic.

Proof. Since $h(\eta(A))=\mathscr{P}(A)$, we need only show that the mapping $\tau$ of the preceding theorem maps $\mathscr{P}(A)$ onto all of $\mathscr{P}(A / \eta(A))$. Now, any member of $\mathscr{P}(A / \eta(A))$ has the form $P / \eta(A)$, where $P$ is a prime ideal in $A$. We claim that $P$ is minimal. For, if $P_{0}$ is any prime ideal in $A$, then $P_{0} \supset \eta(A)$, and if in addition $P_{0} \subset P$, then $P_{0} / \eta(A) \subset P / \eta(A)$. But since the last ideal is minimal, we have $P_{0} / \eta(A)=P / \eta(A)$, whence, $P_{0}=P$. Therefore, $P$ is minimal, and evidently $\tau(P)=P / \eta(A)$.

By virtue of this theorem, no generality will be lost in the study of topological properties of $\mathscr{P}(A)$ if we assume that $A \neq \eta(A)=(0)$. For such rings, Lemma 1.1 takes on the simpler form: $A$ prime ideal $P$ in a ring $A$ without nonzero nilpotents is minimal if and only if for each $x \in P$ there exists $a \in A \sim P$ such that $a x=0$.

If $S \subset A$, we call the set

$$
\mathscr{A}(S)=\{a \in A: a S=0\}
$$

the annihilator of $S$.

2.3. THEOREM. For each element $a$ in a ring without nonzero nilpotents, $h(\mathscr{A}(a))=\mathscr{P} \sim h(a)$. In particular, $h(a)$ and $h(\mathscr{A}(a))$ are disjoint openand-closed sets.

Proof. If $P \in h(a)$, then according to Lemma 1.1, $\mathscr{A}(a)$ is not contained in $P$, i.e., $P \notin h(\mathscr{A}(a))$. Therefore, $h(a) \cap h(\mathscr{A}(a))=\emptyset$. On the other hand, if $P \in \mathscr{P} \sim h(a)$, then for any $x \in \mathscr{A}(a)$, we have $a x=0 \in P$. Since $a \notin P$ and $P$ is prime, $x \in P$. Thus $\mathscr{A}(a) \subset P$, i.e., $P \in h(\mathscr{A}(a))$. Therefore, $h(\mathscr{A}(a))=\mathscr{P} \sim h(a)$. Both sets $h(a)$ and $h(\mathscr{A}(a))$ are closed, and since they are complementary, they are also open.

2.4. Corollary. $P$ is a Hausdorff space with a base of open-and-closed sets.

Proof. Given $P \neq P^{\prime} \in \mathscr{P}$, let $a \in P \sim P^{\prime}$. Then $h(a)$ and $h(\mathscr{A}(a))$ are disjoint open sets containing $P$ and $P^{\prime}$, respectively. Hence $\mathscr{P}$ is a Hausdorff space. As was remarked earlier, the family $\{h(a)\}$ is a base for the closed sets, so that $\{h(\mathscr{A}(a))\}$ is a base for the open sets. And each member of the latter base is closed.

REMARK. Although the family $\{h(a): a \in A\}$ is a base for the closed sets in $\mathscr{P}$ and each member is also open, this family is not generally a base for the open sets. Example 5.7 will substantiate this contention.

2.5. Corollary. An element in a ring without nonzero nilpotents belongs to some minimal prime ideal if and only if it is a divisor of zero. 
Proof. Corollary 1.2 states necessity of the condition (even in a ring with nonzero nilpotents). To prove sufficiency, consider an element $a$ that belongs to no minimal prime ideal. Then $h(a)=\varnothing$ so that $h(\mathscr{A}(a))=\mathscr{P}$. Thus, $\mathscr{A}(a) \subset \cap \mathscr{P}=\eta(A)=(0)$. Therefore, $a$ is not a divisor of zero.

We turn now to consideration of another class of ideals $I$ for which the mapping $\tau$ of Theorem 2.1 is onto all of $\mathscr{P}(A / I)$. These are the ideals $\mathscr{A}(a)$, $a \in A$. We begin by developing some properties of ideals that are annihilators of subsets of $A$.

2.6. Lemma. For any set $S$ in a ring $A$ without nonzero nilpotents, the residue class ring $A / \mathscr{A}(S)$ has no nonzero nilpotent.

Proof. A nilpotent element in the ring $A / \mathscr{A}(S)$ has the form $b+\mathscr{A}(S)$, where $b \in A$ and $b^{n} \in \mathscr{A}(S)$ for some $n \in N$. The condition on $b^{n}$ means that $b^{n} s=0$ for all $s \in S$, which implies $(b s)^{n}=\left(b^{n} s\right) s^{n-1}=0$. Since $A$ has no nonzero nilpotent, $b s=0$ for all $s \in S$; that is, $b \in \mathscr{A}(S)$. Therefore, $b+\mathscr{A}(S)$ is the zero of $A / \mathscr{A}(S)$.

2.7. Theorem. For any set $S$ in a ring $A$ without nonzero nilpotents,

$$
\mathscr{A}(S)=k h(\mathscr{A}(S)) \text {. }
$$

Proof. Certainly, $\mathscr{A}(S) \subset k h(\mathscr{A}(S))$. We reverse this inclusion by producing, for each given $a \in A \sim \mathscr{A}(S)$, a minimal prime ideal $P \in h(\mathscr{A}(S))$ that does not contain $a$.

For the given $a$, there exists $s \in S$ such that $a s \neq 0$. We assert that $a s \notin \mathscr{A}(S)$; since otherwise, $a s^{2}=0$ so that $(a s)^{2}=0$ and hence $a s=0$. It follows from the lemma that the nonzero element as $+\mathscr{A}(S)$ in the ring $A / \mathscr{A}(S)$ fails to belong to some minimal prime ideal, and such an ideal has the form $P / \mathscr{A}(S)$, where $P$ is a prime ideal in $A$. Clearly as $\notin P$, whence $a \notin P$ and $s \notin P$. We complete the proof by showing that $P$ is minimal. Consider any $x \in P$. Since $P / \mathscr{A}(S)$ is a minimal prime ideal in the ring $A / \mathscr{A}(S)$ which has no nonzero nilpotent, there exists $b \in A \sim P$ such that $x b \in \mathscr{A}(S)$. This implies that $x b s=0$. But $b s \notin P$ because $b \notin P$ and $s \notin P$. Consequently, $P$ is minimal.

In order to pass from the hypothesis that $P / \mathscr{A}(S)$ is minimal to the conclusion that $P$ is minimal, we had to produce an element $s$ belonging to $S$ but not to $P$. The existence of such an $s$ depended upon the particular choice of the ideal $P / \mathscr{A}(S)$. In general, if $P$ is a prime ideal containing $\mathscr{A}(S)$ and if $P / \mathscr{A}(S)$ is a minimal prime ideal, we cannot conclude that $P$ is minimal, as is shown by Example 5.7. In other words, it may happen that $P / \mathscr{C}(S)$ is minimal and yet $P$ may contain all of $S$ along with $\mathscr{A}(S)$. But this cannot happen in case $S$ consists of a single element $s$. For, suppose that $a$ is an element of $A$ such that $a s \in \mathscr{A}(s)$. Then $(a s)^{2}=\left(a s^{2}\right) a=0$, whence $a s=0$, so that $a \in \mathscr{A}(s)$. This means that the element $s+\mathscr{A}(s)$ in the ring 
$A / \mathscr{A}(s)$ is a nondivisor of zero, and so it does not belong to the minimal prime ideal $P / \mathscr{A}(s)$. Therefore, $s \notin P$. As a consequence, we have the following lemma.

2.8. Lemma. If a prime ideal $P$ in a ring $A$ without nonzero nilpotents contains a set $\mathscr{A}(s)$ for some $s \in A$ and if $P / \mathscr{A}(s)$ is minimal in the ring $A / \mathscr{A}(s)$, then $P$ is minimal in $A$.

2.9. Theorem. For any element $s$ in a ring $A$ without nonzero nilpotents, the mapping $\tau: h(\mathscr{A}(s)) \rightarrow \mathscr{P}(A / \mathscr{A}(s))$ defined by $\tau(P)=P / \mathscr{A}(s)$ is a homeomorphism of $h(\mathscr{A}(s))$ onto $\mathscr{P}(A / \mathscr{A}(s))$.

Proof. The lemma asserts that the mapping is onto, and Theorem 2.1 that it is a homeomorphism.

Example 5.7, which shows that the lemma would not be valid if the element $s$ were replaced by an arbitrary subset of $A$, shows that such an extension of the theorem would not be valid either.

We have found that the minimal prime ideals of a ring $A$ containing a given ideal $I$ correspond to some of the minimal prime ideals of the ring $A / I$. We show next that the remaining minimal prime ideals of $A$ correspond to all the minimal prime ideals of the ring $I$.

2.10. Theorem. Let $I$ be an ideal in a ring $A$. The mapping $\sigma$ defined by

$$
\sigma(P)=P \cap I, \quad P \in \mathscr{P}(A) \sim h(I),
$$

is a homeomorphism of $\mathscr{P}(A) \sim h(I)$ onto $\mathscr{P}(I)$.

Proof. First we must show that for any $P \in \mathscr{P}(A) \sim h(I)$, the set $P \cap I$, which is obviously a prime ideal in $I$, is minimal. Consider any $x \in P \cap I$. Since $P$ is a minimal prime ideal, there exists $a \in A \sim P$ such that $a x$ is nilpotent. Furthermore, there is an element $b \in I \sim P$, because $P \notin h(I)$. Then $a b \in I \sim(P \cap I)$ because $I$ is an ideal in $A$ while $P$ is a prime ideal containing neither $a$ nor $b$. Since $(a b) x$ is nilpotent, the prime ideal $P \cap I$ of $I$ is minimal.

If $P_{1}$ and $P_{2}$ are distinct members of $\mathscr{P}(A) \sim h(I)$, select $a \in P_{1} \sim P_{2}$ and $b \in I \sim P_{2}$. Then $a b \in I \cap P_{1}$, but $a b \notin P_{2}$. Hence $\sigma\left(P_{1}\right) \neq \sigma\left(P_{2}\right)$.

Next, we show that $\sigma$ maps $\mathscr{P}(A) \sim h(I)$ onto all of $\mathscr{P}(I)$. Consider any $Q \in \mathscr{P}(I)$, and let $J$ be the ideal in $A$ that is generated by $Q$. We assert that $I \cap J=Q$. For, every member of $J$ has the form

$$
\dot{x}=c_{1}+a_{2} c_{2}+\cdots+a_{n} c_{n},
$$

where $a_{i} \in A$ and $c_{i} \in Q$. Since $Q \in \mathscr{P}(I)$, there exist $b_{i} \in I \sim Q, i=1, \cdots$, $n$, such that $b_{i} c_{i}$ is nilpotent. Then $b_{1} b_{2} \cdots b_{n} x$ is also nilpotent. If $x \in I \cap J$, then since $Q$ is prime and $b_{1}, \cdots, b_{n} \notin Q$, we must have $x \in Q$. Thus,

$$
I \cap J \subset Q \text {, }
$$


and so these sets are actually equal. Now, the set $I \sim Q$ is a multiplicative system in $A$ and is disjoint from the ideal $J$. So $J$ is contained in a prime ideal $P^{\prime}$ of $A$, disjoint from $I \sim Q$. In turn, $P^{\prime}$ contains a minimal prime ideal $P$. Then $P \cap I$ is a prime ideal in $I$, and since it is contained in the minimal prime ideal $Q$, we have $P \cap I=Q$.

The proof that $\sigma$ is a homeomorphism is standard; see [4, Theorem 3.1].

The foregoing theorem serves to describe what happens when a unity is adjoined to a ring $A$. Let $A^{\prime}=\{(a, n): a \in A, n \in Z\}$, where $Z$ is the ring of integers, and as usual,

$$
(a, m)+(b, n)=(a+b, m+n) ;(a, m)(b, n)=(a b+m b+n a, m n) .
$$

The ring $A^{\prime}$ has as unity the element $(0,1)$, and $A$ is a prime ideal in $A^{\prime}$. Thus, either $A$ is a minimal prime ideal in $A^{\prime}$ or the hull of $A$ in $A^{\prime}$ is empty.

2.11. Corollary. $\mathscr{P}(A)$ is homeomorphic either with all of $\mathscr{P}\left(A^{\prime}\right)$ or with the complement of a single point in $\mathscr{P}\left(A^{\prime}\right)$.

Either situation described in the corollary can occur. If $A$ is the ring of polynomials over $Z$ with zero constant term, then $A^{\prime}$ is the ring of all polynomials over $Z$ and $h(A)$ is empty. Indeed, $\mathscr{P}(A)=\mathscr{P}\left(A^{\prime}\right)=\{(0)\}$ here.

The second case occurs, for example, whenever the ring $A$ already has a unity element $e$. Then $A$ is a minimal prime ideal in $A^{\prime}$, since $a(1-e)=0$ for all $a \in A$. In this example, the additional point is isolated, for it is $h(e)$.

For an example where the additional point is not isolated, let $A$ be the weak (discrete) direct sum of countably many copies of the integers. $A$ may be regarded as the ring of all sequences of integers that are ultimately zero, and $A^{\prime}$ as the ring of all sequences of integers that are ultimately constant. It is easy to verify that for each positive integer $n$, the set $P_{n}$ (resp. $P_{n}^{\prime}$ ) of all sequences in $A$ (resp. $A^{\prime}$ ) whose $n$th term is 0 is a minimal prime ideal of $A$ (resp. $A^{\prime}$ ). In each space $\mathscr{P}$, these minimal prime ideals are isolated points, because they coincide with $h\left(\mathscr{A}\left(a_{n}\right)\right)$, where $a_{n}$ is the sequence whose $n$th term is 1 and whose remaining terms are 0 . It is not hard to see that the ideals $P_{n}$ constitute all of $\mathscr{P}(A)$. Thus $\mathscr{P}(A)$ is a countable, discrete space. The space $\mathscr{P}\left(A^{\prime}\right)$ consists of the countable discrete set $\left\{P_{n}^{\prime}\right.$ : $n \in N\}$ together with the minimal prime ideal $A$ of $A^{\prime}$. We assert that the neighborhoods of $A$ in $\mathscr{P}\left(A^{\prime}\right)$ are the complements of finite sets. In fact, if

$$
A \in h(\mathscr{A}(a)),
$$

then $a \in A^{\prime} \sim A$, so that the sequence $a$ is ultimately a nonzero constant. If all terms of $a$ past the $m$ th are nonzero, then $h(\mathscr{A}(a)) \supset\left\{P_{n}^{\prime}: n>m\right\}$, i.e., $h(\mathscr{A}(a))$ is the complement of a finite set in $\mathscr{P}\left(A^{\prime}\right)$. This shows that the space $\mathscr{P}\left(A^{\prime}\right)$ is the one-point compactification of the embedded image of the space $\mathscr{P}(A)$. 
3. Compact spaces of minimal prime ideals. Traditionally, compactness of a space of ideals in a ring is associated with the presence of a unity. In the case of the space of minimal prime ideals, however, presence of a unity in the ring has nothing whatever to do with compactness. The relevant condition is that each element $x$ in the ring have a complement $x^{\prime}$ in the sense that $\mathscr{A}\left(x^{\prime}\right)=\mathscr{A}(x)$. We shall show that this condition is sufficient for compactness and almost show that it is necessary. In the proof of necessity, we need an additional hypothesis: Either that the family of closed sets $h(a)$ is closed under finite intersection or that this family is a base for the open sets in $\mathscr{P}$. We suspect that any additional hypothesis is redundant.

There is a close connection between the annihilators of elements and the hulls of the elements as is seen from Theorem 2.3. The next lemma provides further connections.

3.1. Lemma. For all $x, y, z$ in a ring without nonzero nilpotents,

(i) $h(x)=h(\mathscr{A} \mathscr{A}(x))$;

(ii) $\mathscr{A} \mathscr{A}(x y)=\mathscr{A} \mathscr{A}(x) \cap \mathscr{A} \mathscr{A}(y)$;

(iii) $\mathscr{A}(z)=\mathscr{A}(x) \cap \mathscr{A}(y)$ if and only if $h(z)=h(x) \cap h(y)$;

(iv) $\mathscr{A} \mathscr{A}(x)=\mathscr{A}(y)$ if and only if $h(x)=h(\mathscr{A}(y))$.

Proof. (i) Since $x \in \mathscr{A} \mathscr{A}(x), h(x) \supset h(\mathscr{A} \mathscr{A}(x))$. Consider any $P \in h(x)$. Since $P$ is a minimal prime ideal containing $x$, there exists $a \in \mathscr{A}(x) \sim P$. Then, for any $z \in \mathscr{A} \mathscr{A}(x)$, we have $a z=0 \in P$, so that $z \in P$. Thus, $\mathscr{A} \mathscr{A}(x) \subset P$, i.e., $P \in h(\mathscr{A} \mathscr{A}(x))$.

(ii) $\mathscr{A} \mathscr{A}(x y) \subset \mathscr{A}(\mathscr{A}(x) \cup \mathscr{A}(y))=\mathscr{A} \mathscr{A}(x) \cap \mathscr{A} \mathscr{A}(y)$. To reverse this inclusion, consider any $s \in \mathscr{A} \mathscr{A}(x) \cap \mathscr{A} \mathscr{A}(y)$. If $a \in \mathscr{A}(x y)$, then $a x \in \mathscr{A}(y)$ so that $s a x=0$, i.e., $s a \in \mathscr{A}(x)$. Then $s^{2} a=0$. This implies $(s a)^{2}=0$, and hence $s a=0$. Therefore, $s \in \mathscr{A} \mathscr{A}(x y)$.

(iii) Since every member of $\mathscr{P}$ is a prime ideal, the hull of the intersection of two ideals is the union of their hulls. Thus, if $\mathscr{A}(z)=\mathscr{A}(x) \cap \mathscr{A}(y)$, then $h(\mathscr{A}(z))=h(\mathscr{A}(x)) \cup h(\mathscr{A}(y))$. By Theorem 2.3, $h(z)=h(x) \cap h(y)$. On the other hand, if $h(z)=h(x) \cap h(y)$, then

$$
h(\mathscr{A}(z))=h(\mathscr{A}(x)) \cup h(\mathscr{A}(y)) \text {, }
$$

whence

$$
k h(\mathscr{A}(z))=k[h(\mathscr{A}(x)) \cup h(\mathscr{A}(y))]=k h(\mathscr{A}(x)) \cap k h(\mathscr{A}(y)) .
$$

The conclusion then follows from Theorem 2.7.

(iv) This follows from (i) and Theorem 2.7.

By virtue of (iii) the condition that the family of sets $h(x)$ be closed under finite intersection can be stated as a condition on annihilators.

3.2. Definition. A commutative ring $A$ without nonzero nilpotents is said to satisfy the annihilator condition, or is called an a.c. ring, if for every $x, y \in A$, there exists $z \in A$ such that 


$$
\mathscr{A}(z)=\mathscr{A}(x) \cap \mathscr{A}(y)
$$

The following facts are easily verified.

(i) Every subdirect sum of totally ordered integral domains is an a.c. ring. (Take $z=x^{2}+y^{2}$.)

(ii) A ring without nonzero nilpotents in which every finitely generated ideal is principal is an a.c. ring. (Let $z$ be any generator of the ideal $(x, y)$.)

(iii) A complete or weak direct sum of a.c. rings is an a.c. ring.

The class of a.c. rings is evidently very large. In fact, we were unable to find a ring without nonzero nilpotents that failed to satisfy the annihilator condition until Harley Flanders provided us with the following example.

3.3. Example (Flanders). Let $K$ be an algebraically closed field and $\Lambda=K \times K \times K$. In the ring $K^{\Lambda}$ of all $K$-valued functions on $\Lambda$, we denote by $F$ the subring of all functions that vanish everywhere except on a finite subset of $\Lambda$. Furthermore, we single out two elements $x, y \in K^{\Lambda}$ defined by

$$
x(a, b, c)=a, \quad y(a, b, c)=b, \quad \text { for all }(a, b, c) \in \Lambda .
$$

Let $A$ be the smallest subring of $K^{\Lambda}$ that contains $x, y, F$, and the constants. The members of $A$ are, then, all the elements in $K^{\Lambda}$ of the form

$$
f+p(x, y) \text {, }
$$

where $f \in F$ and $p$ is a polynomial in two variables with coefficients in $K$.

The ring $A$ has a unity (the constant 1 ) and no nilpotent except 0 . And it does not satisfy the annihilator condition. For,

$$
\mathscr{A}(x) \cap \mathscr{A}(y)=\{u \in A: u(a, b, c)=0 \text { if } a \neq 0 \text { or } b \neq 0\} .
$$

Suppose that $z \in A$ and $\mathscr{A}(z)=\mathscr{A}(x) \cap \mathscr{A}(y)$. Then $z(a, b, c)=0$ if and only if $a=b=0$. With $z=f+p(x, y)$ as in $\left({ }^{*}\right)$, we have

$$
z(a, b, c)=f(a, b, c)+p(a, b) .
$$

Since the field $K$ is algebraically closed, either the polynomial $p$ is a constant or it vanishes for infinitely many pairs $(a, b)$. In case $p$ is a nonzero constant,

$$
z(0,0, c)=f(0,0, c)+p(0,0)=p(0,0) \neq 0
$$

for all but finitely many $c$ 's, by definition of $F$. In case $p(a, b)=0$ for infinitely many pairs $(a, b)$, then $z(a, b, c)=0$ for infinitely many pairs $(a, b)$. In either case, $z$ cannot satisfy the requirement that $z(a, b, c)=0$ if and only if $a=b=0$.

We come now to the main result of the section.

3.4. Theorem. The following conditions on a ring $A$ without nonzero nilpotents are equivalent: 
(a) $\mathscr{P}(A)$ is compact and $A$ satisfies the annihilator condition.

(b) $\mathscr{P}(A)$ is compact and the family of sets $\{h(a): a \in A\}$ is a base for the open sets of $\mathscr{P}(A)$.

(c) For each $x \in A$ there exists $x^{\prime} \in A$ such that $\mathscr{A} \mathscr{A}\left(x^{\prime}\right)=\mathscr{A}(x)$.

REMarK. As was mentioned earlier, the element $x^{\prime}$ of (c) may be regarded as a (nonunique) complement of $x$. Since $x^{\prime} \in \mathscr{A} \mathscr{A}\left(x^{\prime}\right)=\mathscr{A}(x)$, we have $x x^{\prime}=0$. Furthermore, since $\mathscr{A}\left(x^{\prime}\right)=\mathscr{A} \mathscr{A}\left(x^{\prime}\right)=\mathscr{A} \mathscr{A}(x)$, the element $x$ is a candidate for $\left(x^{\prime}\right)^{\prime}$. The duality between $x$ and $x^{\prime}$ is brought out more clearly by the following characterization of $x^{\prime}: x x^{\prime}=0$ and

$$
\mathscr{A}(x) \cap \mathscr{A}\left(x^{\prime}\right)=(0) \text {. }
$$

The theorem will be proved by means of a sequence of lemmas, the first of which is a strengthened form of the statement "(a) implies (c)."

3.5. Lemma. If $x$ belongs to an a.c. ring $A$ and $h(x)$ is compact, then there exists $x^{\prime} \in \mathscr{A}(x)$ such that $\mathscr{A} \mathscr{A}\left(x^{\prime}\right)=\mathscr{A}(x)$.

Proof. By Theorem 2.3, the set

$$
\bigcap\{h(y) \cap h(x): y \in \mathscr{A}(x)\}=h(\mathscr{A}(x)) \cap h(x)
$$

is empty. Since $h(x)$ is compact and each $h(y)$ is closed, there exist

$$
y_{1}, \cdots, y_{n} \in \mathscr{A}(x)
$$

such that

$$
h\left(y_{1}\right) \cap \cdots \cap h\left(y_{n}\right) \cap h(x)=\emptyset .
$$

This implies that

$$
h\left(y_{1}\right) \cap \cdots \cap h\left(y_{n}\right)=h(\mathscr{A}(x)) .
$$

Since $A$ is an a.c. ring, there exists (by induction) an element $x^{\prime} \in A$ such that

$$
\mathscr{A}\left(x^{\prime}\right)=\mathscr{A}\left(y_{1}\right) \cap \cdots \cap \mathscr{A}\left(y_{n}\right) .
$$

By Lemma 3.1 (iii) we have

$$
h\left(x^{\prime}\right)=h\left(y_{1}\right) \cap \cdots \cap h\left(y_{n}\right)=h(\mathscr{A}(x)),
$$

and by Lemma 3.1 (iv), $\mathscr{A} \mathscr{A}\left(x^{\prime}\right)=\mathscr{A}(x)$. As was remarked above, this implies that $x^{\prime} \in \mathscr{A}(x)$.

A similarly strengthened form of the statement "(b) implies (c)" is also available:

3.6. Lemma. If the family of sets $\{h(a): a \in A\}$ is a base for the open sets in $\mathscr{P}(A)$ and if $x$ is an element of $A$ for which $h(\mathscr{A}(x))$ is compact, then there exists $x^{\prime} \in \mathscr{A}(x)$ such that $\mathscr{A} \mathscr{\mathscr { A }}\left(x^{\prime}\right)=\mathscr{A}(x)$. 
Proof. By hypothesis, the open set $h(\mathscr{A}(x))$ is a union of open sets of the form $h(a)$, and since $h(\mathscr{A}(x))$ is also compact, it is a finite union:

$$
h(\mathscr{A}(x))=h\left(a_{1}\right) \cup h\left(a_{2}\right) \cup \cdots \cup h\left(a_{n}\right) .
$$

Let $x^{\prime}=a_{1} a_{2} \ldots a_{n}$. Then $h\left(x^{\prime}\right)=h\left(a_{1}\right) \cup h\left(a_{2}\right) \cup \cdots \cup h\left(a_{n}\right)=h(\mathscr{A}(x))$. As before, this implies $\mathscr{A} \mathscr{A}\left(x^{\prime}\right)=\mathscr{A}(x)$ and $x^{\prime} \in \mathscr{A}(x)$.

3.7. Corollary. Under conditions (a) or (b) of the theorem, the ring A contains a nondivisor of zero.

Proof. An element $0^{\prime}$ as given by either of the lemmas is a nondivisor of zero, because $\mathscr{A} \mathscr{A}\left(0^{\prime}\right)=\mathscr{A}(0)=A$, so that $\mathscr{A}\left(0^{\prime}\right)=\mathscr{A} \mathscr{A}(0)=(0)$.

Now we turn to the proof that (c) implies (a) and (b). To begin with, (c) implies the annihilator condition: simply set $z=\left(x^{\prime} y^{\prime}\right)^{\prime}$. For, using Lemma 3.1 (ii), we have

$$
\begin{aligned}
\mathscr{A}(z) & =\mathscr{A} \mathscr{A} \mathscr{A}(z)=\mathscr{A} \mathscr{A}\left(x^{\prime} y^{\prime}\right)=\mathscr{A} \mathscr{A}\left(x^{\prime}\right) \cap \mathscr{A} \mathscr{A}\left(y^{\prime}\right) \\
& =\mathscr{A}(x) \cap \mathscr{A}(y) .
\end{aligned}
$$

Furthermore, we know that the family of sets $\{h(\mathscr{A}(x)): x \in A\}$ is a base for the open sets of $\mathscr{P}(A)$. Under condition (c), $h(\mathscr{A}(x))=h\left(\mathscr{A} \mathscr{A}\left(x^{\prime}\right)\right)$, which is $h\left(x^{\prime}\right)$ by Lemma 3.1 (i). Thus, condition (c) implies that the family $\{h(a): a \in A\}$ is a base for the open sets of $\mathscr{P}(A)$. All that remains is to prove that $\mathscr{P}(A)$ is compact.

We saw earlier (Corollary 2.5) that in a ring without nonzero nilpotents an element belongs to some minimal prime ideal if and only if it is a divisor of zero. Our next lemma is an analogous statement about ideals in a ring that satisfies condition (c).

3.8. Lemma. Let A satisfy condition (c). An ideal I of $A$ is contained in some minimal prime ideal of $A$ if and only if every member of $I$ is a divisor of 0 . In particular, a prime ideal in $A$ is minimal if and only if each of its members is a divisor of 0 .

Proof. We have observed that the condition is necessary.

Conversely, let every member of $I$ be a divisor of 0 . The set $M$ of all nondivisors of 0 in $A$ is a multiplicative system disjoint from $I$. Hence $I$ is contained in a prime ideal $P$ that is disjoint from $M$; that is, every member of $P$ is a divisor of 0 . We shall prove that $P$ is minimal. Given $x \in P$, let $x^{\prime}$ be as in condition (c). Then

$$
h\left(x^{\prime}\right)=h\left(\mathscr{A} \mathscr{A}\left(x^{\prime}\right)\right)=h(\mathscr{A}(x))=\mathscr{P} \sim h(x) .
$$

This means that each minimal prime ideal contains exactly one of $x$ and $x^{\prime}$. Hence, $h\left(x+x^{\prime}\right)=\emptyset$, so by Corollary 2.5, $x+x^{\prime}$ is not a divisor of 0 . Thus, $x+x^{\prime} \notin P$, whence $x^{\prime} \notin P$. Since $x^{\prime} \in \mathscr{A}(x)$, we conclude that $P$ is minimal. 
To complete the proof that $\mathscr{P}(A)$ is compact, let $\left\{h\left(x_{\alpha}\right)\right\}$ be a family of closed sets in $\mathscr{P}$ with empty intersection. We want to produce a finite subfamily with empty intersection. Let $I$ be the smallest ideal in $A$ containing all of the elements $x_{\alpha}$. Then

$$
h(I)=\bigcap_{\alpha} h\left(x_{\alpha}\right)=\emptyset .
$$

By Lemma 3.8, I contains a nondivisor of zero-call it $e$. There exist $x_{\alpha_{i}}$, $a_{i} \in A$, and $k_{i} \in N, i=1, \cdots, n$, such that $e=\sum_{1}^{n} a_{i} x_{\alpha_{i}}+\sum_{1}^{n} k_{i} x_{\alpha_{i}}$. And

$$
\bigcap_{i=1}^{n} h\left(x_{\alpha_{i}}\right) \subset h(e)=\emptyset,
$$

which provides the desired finite subfamily.

A zero-set in a topological space $X$ is a set of the form $\{x \in X: f(x)=0\}$ for some continuous real-valued function $f$ on $X$.

3.9. Corollary. If $A$ is an a.c. ring and $\mathscr{P}(A)$ is compact, then every zero-set in $\mathscr{P}(A)$ is a countable intersection of hulls of elements of $A$.

Proof. Any zero-set $\mathscr{F}$ of $\mathscr{P}(A)$ is a compact $G_{\delta}$, that is, there exists a sequence $\left\{\mathscr{U}_{n}\right\}$ of open sets in $\mathscr{P}(A)$ such that $\mathscr{Z}=\bigcap_{n=1}^{\infty} \mathscr{U}_{n}$. By the theorem, each $\mathscr{U}_{n}$ is a union of hulls of elements of $A$, and since $\mathscr{Z}$ is compact, it is contained in a finite union of such hulls. Thus, for each fixed $n$, there exist $u_{1}, \cdots, u_{k} \in A$ such that

$$
\mathscr{Z} \subset \bigcup_{i=1}^{k} h\left(u_{i}\right) \subset \mathscr{U}_{n}
$$

Let $a_{n}=\prod_{i=1}^{k} u_{i}$. Then $\mathscr{Z}=\bigcap_{n=1}^{\infty} h\left(a_{n}\right)$.

3.10. Remarks. (i) As we mentioned in the first paragraph of $\S 3$, we do not know whether a ring without nonzero nilpotents whose space of minimal prime ideals is compact is necessarily an a.c. ring. The ring $A$ of Example 3.3 does not satisfy the annihilator condition, but also, the space $\mathscr{P}(A)$ is not compact. We shall show, in fact, that $\mathscr{P}(A)$ is not even countably compact (cf. Theorem 4.9).

For each $\lambda \in \Lambda$, the set

$$
P_{\lambda}=\{u \in A: u(\lambda)=0\}
$$

is obviously a prime ideal in $A$, and these ideals are distinct for distinct $\lambda$. Moreover, the element $v_{\lambda} \in F$ that is 1 at $\lambda$ and 0 elsewhere belongs to $\mathscr{A}\left(P_{\lambda}\right) \sim P_{\lambda}$. Hence, $P_{\lambda}$ is minimal.

If $a \in A$ does not belong to $P_{\lambda}$, then $a v_{\lambda}$ is a nonzero constant multiple of $v_{\lambda}$. Hence, $v_{\lambda}$ belongs to any ideal that is not contained in $P_{\gamma}$, and any ideal that is contained in none of the $P_{\lambda}$ 's must contain all of the $v_{\lambda}$ 's, and so it must contain $F$. Now, $F$ itself is a prime ideal in $A$. An ideal that is 
contained properly in $F$ is contained in some $P_{\lambda}$ and cannot be prime. Therefore, $F$ is a minimal prime ideal and

$$
\mathscr{P}(A)=\left\{P_{\lambda}: \lambda \in \dot{\epsilon} \Lambda \cup\{F\} .\right.
$$

Each $P_{\lambda}$ is an isolated point of $\mathscr{P}$ because $\left\{P_{\lambda}\right\}=h\left(1-v_{\lambda}\right)$. Since $x \notin F$, the set $h(x)$ coincides with $\left\{P_{\lambda}: \lambda=(0, b, c)\right\}$, which is an infinite, closed, discrete set in $\mathscr{P}$. Thus, $\mathscr{P}$ is not countably compact.

(ii) The proof of Lemma 3.5 shows that if $A$ has no nonzero nilpotent and $\mathscr{P}(A)$ is compact, then for each $x \in A$ there exist $y_{1}, \cdots, y_{n}$ such that $h(\mathscr{A}(x))=h\left(y_{1}\right) \cap \cdots \cap h\left(y_{n}\right)$, or equivalently (as in the proof of Lemma 3.1 (iii)), $\mathscr{A}(x)=\mathscr{A}\left(y_{1}\right) \cap \cdots \cap \mathscr{A}\left(y_{n}\right)$. We do not know whether this necessary condition for compactness of $\mathscr{P}(A)$ is also sufficient.

\section{Countably compact and basically disconnected spaces of minimal prime ideals.}

4.1. Definition. A ring $A$ without nonzero nilpotents is said to satisfy the countable annihilator condition, or is called a c.a.c. ring, if for each sequence $\left\{x_{n}\right\}$ in $A$, there is an $x \in A$ such that $\mathscr{A}(x)=\bigcap_{n=1}^{\infty} \mathscr{A}\left(x_{n}\right)$.

Obviously, every c.a.c. ring is an a.c. ring. The weak direct sum of denumerably many copies of the real field is an example of an a.c. ring that is not a c.a.c. ring. To see the latter, let $\left\{x_{n}\right\}$ be the sequence of all elements in the ring, one of whose coordinates is 1 and the remainder 0 . Then

$$
\bigcap_{n=1}^{\infty} \mathscr{A}\left(x_{n}\right)=(0),
$$

while every element in the ring is a proper divisor of 0 .

If $\left\{x_{n}\right\}$ is a sequence in the ring $C(X)$ of all continuous real-valued functions on a topological space $X$ (or, more generally, in a uniformly closed $\Phi$-algebra in the sense of $[3])$, and $x=\sum_{n=1}^{\infty} 2^{-n}\left(\left|x_{n}\right| \wedge 1\right)$, then

$$
\mathscr{A}(x)=\bigcap_{n=1}^{\infty} \mathscr{A}\left(x_{n}\right)
$$

In other words, $C(X)$ is always a c.a.c. ring. Also, a ring without nonzero nilpotents in which every countably generated ideal is principal is a c.a.c. ring.

4.2. Lemma. If $B$ is a subset of a ring without nonzero nilpotents and $\mathscr{S}=\bigcup\{h(\mathscr{A}(b)): b \in B\}$, then the closure of $\mathscr{S}$ is $h(\mathscr{A}(B))$.

Proof. $\quad k(\mathscr{S})=\bigcap\{k h(\mathscr{A}(b)): b \in B\}=\bigcap\{\mathscr{A}(b): b \in B\}=\mathscr{A}(B)$, by Theorem 2.7. Hence, cl $\mathscr{S}=h k(\mathscr{S})=h(\mathscr{A}(B))$.

A topological space $X$ is said to be extremally disconnected if the closure of every open set in $X$ is open. $X$ is basically disconnected if the closure of the complement of each zero-set in $X$ is open. It is well known that $C(X)$ is con- 
ditionally complete (resp. conditionally $\sigma$-complete) as a lattice if and only if $X$ is extremally (resp., basically) disconnected [2, p.52].

4.3. LemMA. For a ring $A$ without nonzero nilpotents, $\mathscr{P}(A)$ is extremally disconnected if and only if $h(\mathscr{A}(B))$ is open for every subset $B$ of $A$.

Proof. Every open subset of $\mathscr{P}$ is a union of hulls of annihilators of elements of $A$, that is, it is of the form $\mathscr{S}$ of the preceding lemma.

4.4. Theorem. Let $A$ be an a.c. ring. $\mathscr{P}(A)$ is compact and extremally disconnected if and only if for each set $B \subset A$ there is $a y \in A$ such that

$$
\mathscr{A}(B)=\mathscr{A}(y) \text {. }
$$

Proof. If such a $y$ exists for each $B$, then $\mathscr{P}(A)$ is compact by Theorem 3.4 (with $B=\mathscr{A}(x)$ and $x^{\prime}=y$ ), and it is extremally disconnected by Lemma 4.3 and Theorem 2.3.

Conversely, assume that $\mathscr{P}$ is compact and extremally disconnected. Then for each set $B \subset A$, the set $h(\mathscr{A}(B))$ is open as well as closed. By Theorem 3.4 , the family $\{h(a): a \in A\}$ is a base for the topology of $\mathscr{P}$; hence, the open set $\mathscr{P} \sim h(\mathscr{A}(B))$ is a union of such basic open sets. Since

$$
\mathscr{P} \sim h(\mathscr{A}(B))
$$

is also compact, it is a union of a finite number of such sets:

$$
\mathscr{P} \sim h(\mathscr{A}(B))=\bigcup_{i=1}^{n} h\left(a_{i}\right)
$$

Let $y=a_{1} a_{2} \ldots a_{n}$. Then,

$$
\mathscr{P} \sim h(\mathscr{A}(B))=\bigcup_{i=1}^{n} h\left(a_{i}\right)=h(y)=\mathscr{P} \sim h(\mathscr{A}(y)) .
$$

Thus, $h(\mathscr{A}(B))=h(\mathscr{A}(y))$, and by Theorem 2.7 , this implies

$$
\mathscr{A}(B)=\mathscr{A}(y) \text {. }
$$

4.5. Theorem. If $A$ is a c.a.c. ring and $\mathscr{P}(A)$ is compact, then $\mathscr{P}(A)$ is basically disconnected.

Proof. By Corollary 3.9 and Lemma 4.2, the closure of the complement of a zero-set in $\mathscr{P}(A)$ is the hull of the annihilator of a countable set in $A$. Since $A$ is a c.a.c. ring, such a set is open.

The example at the end of $\$ 2$ shows that an a.c. ring may have a compact space of minimal prime ideals that is not basically disconnected. We shall see (Theorem 4.7) that if $A$ is a c.a.c. ring and $\mathscr{P}(A)$ is only locally compact, then $\mathscr{P}(A)$ is also basically disconnected. In fact, we have not been able to find a c.a.c. ring $A$ for which $\mathscr{P}(A)$ is not basically disconnected. Example 5.10 provides a c.a.c. ring $A$ for which $\mathscr{P}(A)$ is basically, but not extremally, disconnected. 
4.6. LEMMA. If the topology of a space $X$ has a base of open-and-closed sets each of which is basically disconnected, then $X$ is basically disconnected.

Proof. Let $Z$ be a zero-set in $X$ and let $x$ be a point in cl int $Z$. Our objective is to prove that $x \in \operatorname{int} Z$. Consider any open-and-closed, basically disconnected set $V$ that contains $x$. Since $V$ is a neighborhood of $x, x$ belongs to $\operatorname{cl}(V \cap \operatorname{int} Z)$. Now $V \cap Z$ is a zero-set in $V$, and hence its interior relative to $V$ is closed. But, since $V$ is open-and-closed, interiors and closures relative to $V$ coincide with interiors and closures, respectively, relative to $X$. Therefore, $V \cap \operatorname{int} Z=\operatorname{int}(V \cap Z)$, and since the latter set is closed,

$$
x \in \operatorname{cl}(V \cap \operatorname{int} Z)=\operatorname{int}(V \cap Z)=V \cap \operatorname{int} Z .
$$

In particular, $x \in$ int $Z$.

4.7. Theorem. If $A$ is a c.a.c. ring and $\mathscr{P}(A)$ is locally compact, then $\mathscr{P}(A)$ is basically disconnected.

Proof. The sets of the form $h(\mathscr{A}(x)), x \in A$, constitute a base for the topology of $\mathscr{P}(A)$, each of which is closed as well as open. Since $\mathscr{P}(A)$ is locally compact, the base $\{h(\mathscr{A}(x)): x \in A\}$ contains a base each of whose members is compact. Now, according to Theorem $2.9, h(\mathscr{A}(x))$ and

$$
\mathscr{P}(A / \mathscr{A}(x))
$$

are homeomorphic. Moreover, by Lemma 2.6, $A / \mathscr{A}(x)$ has no nonzero nilpotent and hence it is a c.a.c. ring. Thus, by Theorem 4.5 , each compact $h(\mathscr{A}(x))$ is basically disconnected. By the preceding lemma, $\mathscr{P}(A)$ is also basically disconnected.

In $\$ 5$, we shall give an example (5.7) of a c.a.c. ring with locally compact but not compact space of minimal prime ideals and one (5.9) whose space of minimal prime ideals is not locally compact. We conclude the present section by proving that the space of minimal prime ideals of a c.a.c. ring is always countably compact:

As before, $\boldsymbol{N}$ denotes the set of positive integers. Recall that an ultrafilter on $N$ is a maximal family of subsets of $N$ with the finite intersection property.

4.8. Lemma. Let $\left\{P_{n}: n \in N\right\}$ be a sequence of minimal prime ideals of a c.a.c. ring $A$, let $U$ be an ultrafilter on $N$, and for each $x \in A$ let $E(x)$ $=\left\{n \in N: x \in P_{n}\right\}$. Then the set

$$
P(U)=\{x \in A: E(x) \in U\}
$$

is a minimal prime ideal of $A$.

Proof. Since $E(x y)=E(x) \cup E(y)$ and $E(x-y) \supset E(x) \cap E(y)$, the set $P(U)$ is an ideal of $A$. Moreover, if $x y \in P(U)$, then

$$
\boldsymbol{E}(x) \cup \boldsymbol{E}(y)=\boldsymbol{E}(x y) \in \boldsymbol{U},
$$


so either $\boldsymbol{E}(x) \in \boldsymbol{U}$, or $\boldsymbol{E}(y) \in U$ because $U$ is an ultrafilter. Therefore, $P(U)$ is prime.

To see that $P(U)$ is minimal, consider any $x \in P(U)$. For each $n \in E(x)$, there is an $a_{n} \in A \sim P_{n}$ such that $x a_{n}=0$. Since $A$ is a c.a.c. ring, there is an $a \in A$ such that $\mathscr{A}(a)=\bigcap_{n \in E(x)} \mathscr{A}\left(a_{n}\right)$. This means that $x a=0$. Moreover, for each such $n, a \notin P_{n}$, because

$$
\mathscr{A}(a) \subset \mathscr{A}\left(a_{n}\right) \subset P_{n}
$$

Thus, $\boldsymbol{E}(a) \cap \boldsymbol{E}(x)=\emptyset$. Since $\boldsymbol{E}(x)$ belongs to the filter $U, E(a) \notin U$. Therefor $a \notin P(U)$, whence $P(U)$ is minimal.

4.9. Theorem. If $A$ is a c.a.c. ring, then $\mathscr{P}(A)$ is countably compact.

Proof. We show that any sequence $\left\{P_{n}: n \in N\right\}$ in $\mathscr{P}(A)$ has a cluster point, namely, any ideal $P(U)$ where $U$ is a free ultrafilter on $N$, i.e., an ultrafilter whose total intersection is empty. For each $j \in N$, the set

$$
\{n \in N: n \geqq j\}
$$

belongs to $U$ because $U$ is free. Hence,

$$
\bigcap_{n \geq j} P_{n} \subset P(U)
$$

Now the set on the left side of this inclusion is precisely $k\left(\left\{P_{n}: n \geqq j\right\}\right)$, and so the inclusion itself is equivalent to the statement: $P(U)$ belongs to the closure of the set $\left\{P_{n}: n \geqq j\right\}$ in $\mathscr{P}(A)$. As this is true for all $j, P(U)$ is a cluster point of the sequence.

5. Function rings. This section is concerned with the special properties of the space of minimal prime ideals of a ring that happens to be the ring $C(X)$ of all continuous real-valued functions on some completely regular space $X$. Most of the results apply equally well to $\Phi$-algebras [3], that is, to archimedean lattice-ordered algebras over the reals with unity elements that are also weak order units. The $\Phi$-algebras are definitely more general than subalgebras of algebras of the form $C(X)$. For example, the Lebesgue measurable functions modulo the ideal of null functions is a $\Phi$-algebra that is not isomorphic to any algebra of real-valued functions.

We begin by showing that the ring $C^{*}(X)$ of all bounded continuous functions on a given space $X$ has a space of minimal prime ideals that is homeomorphic with that of $C(X)$. With a view toward possible application to $\Phi$ algebras, we cast the result in a general setting.

5.1. TheOREM. Let $A$ be a subring of a commutative ring $B$ without nonzero nilpotents, and suppose that for each $b \in B$, there exist $a_{b} \in A$ and $u_{b} \in B$ such that $b=a_{b} u_{b}$ and $\mathscr{A}\left(u_{b}\right)=(0)$. Then the mapping $\sigma$ defined by 


$$
\sigma(P)=P \cap A, \quad P \in \mathscr{P}(B),
$$

is a homeomorphism of $\mathscr{P}(B)$ onto $\mathscr{P}(A)$.

Proof. To begin with, if $P \in \mathscr{P}(B)$, then $P \cap A$ is a prime ideal of $A$. Moreover, if $x \in P \cap A$, then there exists $b \in B \sim P$ such that $x b=0$. Then, $x a_{b} u_{b}=0$, and since $\mathscr{A}\left(u_{b}\right)=(0)$, we have $x a_{b}=0$. Also, $a_{b} \notin P$ because $b \notin P$. Therefore, $P \cap A$ is a minimal prime ideal in $A$. Thus, $\sigma$ is a mapping from $\mathscr{P}(B)$ into $\mathscr{P}(A)$. It is clearly continuous.

To prove that $\sigma$ has the other stated properties, consider any $Q \in \mathscr{P}(A)$ and let $I$ be the smallest ideal in $B$ that contains $Q$.

$$
\text { If } y \in I \text {, there exists } z \in A \sim Q \text { such that } y z=0 \text {. }
$$

For, $y \in I$ implies $y=q_{0}+q_{1} b_{1}+\cdots+q_{n} b_{n}$ for some $q_{0}, q_{1}, \cdots, q_{n} \in Q$ and $b_{1}, \cdots, b_{n} \in B$. Since $Q$ is a minimal prime ideal in $A$, there exist $z_{i} \in A \sim Q$, $i=0, \cdots, n$, such that $q_{i} z_{i}=0$. Then, $y z=0$ with $z=z_{0} z_{1} \cdots z_{n} \in A \sim Q$.

Now, $I \cap A \supset Q$, trivially. To reverse this inclusion, consider $y \in I \cap A$. Since $y z=0$ for some $z \in A \sim Q$ and $Q$ is a prime ideal, $y \in Q$.

To prove that $I$ is a prime ideal in $B$, consider any $b_{1}, b_{2}$ such that $b_{1} b_{2} \in I$ and select $z \in A \sim Q$ so that $b_{1} b_{2} z=0$. Then $a_{b_{1}} u_{b_{1}} a_{b_{2}} u_{b_{2}} z=0$. Since $\mathscr{A}\left(u_{b_{1}} u_{b_{2}}\right)=(0)$, we have $a_{b_{1}} a_{b_{2}} z=0$. Hence one of $a_{b_{1}}, a_{b_{2}}$ belongs to the prime ideal $Q$, and the corresponding $b_{i}$ belongs to the ideal $I$. Therefore $I$ is prime. It is minimal by $\left(^{*}\right)$.

So far, we have proved that $\sigma$ is a continuous mapping from $\mathscr{P}(B)$ onto $\mathscr{P}(A)$. It is obviously one-one. Moreover, $\sigma[h(b)]=h\left(a_{b}\right)$ for all $b \in B$, whence $\sigma^{-1}$ is continuous.

5.2. Corollary. For any space $X, \mathscr{P}(C(X))$ and $\mathscr{P}\left(C^{*}(X)\right)$ are homeomorphic.

Proof. Apply the theorem with $A=C^{*}(X), B=C(X), a_{b}=(-1)$ $\vee(b \wedge 1)$, and $u_{b}=|b| \vee 1$.

Since $C^{*}(X)$ is isomorphic with $C(\beta X)$, there would be no loss of generality in the study of topological properties of $\mathscr{P}(C(X))$, according to this corollary, if we were to assume that $X$ is compact. This assumption would affect the relations between the space $\mathscr{P}(C(X))$ and the space $X$, however; we shall therefore avoid it.

We recall [2] that every prime ideal in $C(X)$ is contained in a unique maximal ideal, and that the maximal ideals are in one-one correspondence with the points $p$ of $\beta X$, given by the formula

$$
\boldsymbol{M}^{p}=\left\{f \in C(X): p \in \operatorname{cl}_{\beta X} \boldsymbol{Z}(f)\right\},
$$

where $Z(f)=\{x \in X: f(x)=0\}$. Thus, we may define a mapping

$$
\text { ı: } \mathscr{P}((C) X) \rightarrow \beta X
$$


by setting $\iota(P)=p$ if $P \subset M^{p}$. This mapping can be described, alternatively, with the aid of the ideals

$$
O^{p}=\left\{f \in C(X): p \in \operatorname{int} \operatorname{cl}_{\beta X} Z(f)\right\} .
$$

Namely, every prime ideal contains exactly one ideal of the form $O^{p}$. For $P \in \mathscr{P}(C), \iota(P)$ is the unique $p \in \beta X$ such that $O^{p} \subset P$.

5.3. ThEOREM. (a) เ is a continuous mapping of $\mathscr{P}(C(X))$ onto $\beta X$.

(b) ı maps no proper closed subset of $\mathscr{P}$ onto $\beta X$.

(c) $\iota$ is one-one if and only if $O^{p}$ is a prime ideal for each $p \in \beta X$, that is, $X$ is an $F$-space.

(d) $\iota$ is a homeomorphism if and only if the interior of every zero-set in $X$ is closed, that is, $X$ is basically disconnected.

(e) If $X$ is an $F$-space, then $\mathscr{P}(C(X))$ is compact if and only if $X$ is basically disconnected.

Proof. (a) Given an open neighborhood $U$ of a point $p$ in $\beta X$, there is a function $g \in C(\beta X)$ that vanishes on some neighborhood of $p$ and is equal to 1 outside of $U$. We claim that if $f$ is the restriction of $g$ to $X$, then

$$
\iota^{-1}(p) \subset h(f) \subset \iota^{-1}[U],
$$

and since $h(f)$ is an open set in $\mathscr{P}(C(X))$, this implies that $\iota$ is continuous. To prove our assertions about $f$, note first that $p \in$ int $\operatorname{cl}_{\beta X} Z(f)$ so that $f \in O^{p}$, whence $h(f) \supset \iota^{-1}(p)$. Secondly, if $f \in M^{q}$ for some $q$ in $\beta X$, then $q \in \operatorname{cl}_{\beta X} Z(f)$ so that $g(q)=0$. Thus, $q \in U$. This means that $\iota[h(f)] \subset U$.

(b) Every proper closed set in $\mathscr{P}$ is contained in a set $h(f)$ for some nonzero $f \in C(X)$, because such sets form a base for the closed sets. Let $p$ be a point in $X$ where $f(p) \neq 0$. Then $f \notin M^{p}$, and this implies $p \notin \iota[h(f)]$.

(c) $O^{p}$ is the intersection of all the minimal prime ideals that are contained in $\boldsymbol{M}^{p}$.

(d) As was observed in [2, Problem 6M, p. 96], $X$ is basically disconnected if and only if $\beta X$ is. Therefore, by Corollary 5.2, we may assume that $X=\beta X$, that is, $X$ is compact. Now, if $\iota$ is one-one, then the minimal prime ideals of $C$ are precisely the ideals $O^{p}, p \in X$. Moreover, for any $f \in C$,

$$
{ }_{\iota}[h(f)]=\left\{p \in X: f \in O^{p}\right\}=\{p \in X: p \in \operatorname{int} \operatorname{cl} Z(f)\}=\operatorname{int} Z(f) .
$$

And if $\iota$ is a homeomorphism, then $\iota[h(f)]$ is a closed set.

Suppose, conversely, that $X$ is compact and basically disconnected. Since every basically disconnected space is an $F$-space $[2,14 \mathrm{~N}$, p. 215], the mapping $\iota$ is one-one, and it is a closed mapping because $\iota[h(f)]=\operatorname{int} Z(f)$ for all $f \in C$.

(e) is an immediate consequence of (c) and (d).

We have already observed in $\$ 4$ that every $C(X)$ satisfies the countable 
annihilator condition. As a consequence, $\mathscr{P}(C(X))$ is always countably compact. It is not always compact, however. According to (e) of the preceding theorem, if $X$ is an $F$-space that is not basically disconnected (see Example $5.9)$, then $\mathscr{P}(C(X))$ is not compact. Now we investigate conditions on $X$ that will make $\mathscr{P}(C(X))$ compact.

5.4. Lemma. For any functions $f, f^{\prime}$ in $C(X)$ :

(a) $h\left(f^{\prime}\right) \supset h(\mathscr{A}(f))$ if and only if $\boldsymbol{Z}(f) \cup \boldsymbol{Z}\left(f^{\prime}\right)=X$;

(b) $h\left(f^{\prime}\right) \subset h(\mathscr{A}(f))$ if and only if $\operatorname{int}\left[\boldsymbol{Z}(f) \cap \boldsymbol{Z}\left(f^{\prime}\right)\right]=\emptyset$.

Proof. (a) Each condition is equivalent to $f^{\prime} \in \mathscr{A}(f)$.

(b) The following statements are equivalent:

$$
\begin{aligned}
\operatorname{int}\left[Z(f) \cap Z\left(f^{\prime}\right)\right] & =\operatorname{int} Z\left(f^{2}+f^{\prime 2}\right)=\emptyset ; \quad \mathscr{A}\left(f^{2}+f^{\prime 2}\right)=(0) ; \\
h\left(f^{2}+f^{\prime 2}\right) & =\emptyset ; \quad h(f) \cap h\left(f^{\prime}\right)=\emptyset ; \quad h\left(f^{\prime}\right) \subset h(\mathscr{A}(f)) .
\end{aligned}
$$

Theorem 3.4 may now be restated for a ring $C(X)$ as follows:

5.5. Corollary. The space $\mathscr{P}(C(X))$ is compact if and only if for each $f \in C(X)$ there exists $f^{\prime} \in C(X)$ such that

$$
Z(f) \cup Z\left(f^{\prime}\right)=X \text { and } \operatorname{int}\left[\boldsymbol{Z}(f) \cap Z\left(f^{\prime}\right)\right]=\emptyset .
$$

The next theorem provides conditions on $X$ that may be easy to verify and that are sufficient to make $\mathscr{P}(C(X))$ have some of the properties studied in $\$ 4$.

5.6. THEOREM. If the support of each function in $C(X)$ is a zero-set, then $\mathscr{P}(C(X))$ is compact and basically disconnected. If the closure of each open set in $X$ is the support of a continuous function, in particular, if $X$ is perfectly normal, then $\mathscr{P}(C(X))$ is compact and extremally disconnected.

Proof. The first part is immediate from the corollary and Theorem 4.5.

To prove the second part, we apply Theorem 4.4. Let $B$ be any subset of $C(X)$, and $S=\bigcap\{Z(f): f \in B\}$. Choose $g$ in $C(X)$ with support $\operatorname{cl}(X \sim S)$. Then $\mathscr{A}(B)=\mathscr{A}(g)$.

5.7. EXAMPLE. A compact space $\Gamma$ such that

(i) $\mathscr{P}(C(\Gamma))$ is locally compact but not compact,

(ii) $\{h(f): f \in C(\Gamma)\}$ is not a base for the topology of $\mathscr{P}(C(\Gamma))$,

(iii) $C(\Gamma)$ has an ideal $S$ and a prime ideal $P$ such that $P / \mathscr{A}(S)$ is minimal in $C / \mathscr{A}(S)$, but $P$ is not minimal in $C$.

Let $W^{*}$ denote the totally ordered space of ordinal numbers less than or equal to the first uncountable ordinal $\omega_{1}$. This space is compact, and if $f \in C\left(W^{*}\right)$ then there is an $\alpha<\omega_{1}$ such that $f(x)=f\left(\omega_{1}\right)$ for all $\alpha \leqq x \leqq \omega_{1}$ $\left[2\right.$, p. 75]. The quotient space of $W^{*}$ obtained by identifying $\omega_{0}$ with $\omega_{1}$ will be denoted $\Gamma$. The identified point of $\Gamma$ will still be called $\omega_{1}$. Clearly, the 
space $\Gamma$ is compact. We define a function $s \in C(\Gamma)$ by letting $s(0)=1$, $s(n)=1 / n$ if $0<n<\omega_{0}, s(x)=0$ if $\omega_{0}<x$.

Let $K=\left\{n \in \Gamma: n<\omega_{0}\right\} \cup\left\{\omega_{1}\right\}$ and $L=\left\{\alpha \in \Gamma: \omega_{0}<\alpha \leqq \omega_{1}\right\}$. Then $K$ and $L$ are compact subsets of $\Gamma, K \cup L=\Gamma, K$ is homeomorphic with the one-point compactification $N^{*}$ of the countable discrete space $N$, and $L$ is homeomorphic with $W^{*}$.

Now, $K$ is the support of $s$ while $L=Z(s)$, but $K$ is not a zero-set. In fact, if $Z(g) \supset K$, or equivalently, if $g \in \mathscr{A}(s)$, then $g\left(\omega_{1}\right)=0$, and there exists $\alpha<\omega_{1}$ such that $\left\{x \in \Gamma: \alpha \leqq x \leqq \omega_{1}\right\} \subset Z(g)$. Thus, $K \neq Z(g)$; moreover, $\operatorname{int}[\boldsymbol{Z}(g) \cap Z(s)] \neq \emptyset$. By Corollary 5.5, $\mathscr{P}(C(\Gamma))$ is not compact. Indeed, $h(s)$ is not compact (Lemma 3.5).

To prove that $\mathscr{P}$ is locally compact, we must find for each $P \in \mathscr{P}$ a function $f \in C(\Gamma)$ such that $P \in h(\mathscr{A}(f))$ (i.e. $f \notin P)$ and $h(\mathscr{A}(f))$ is compact. According to Theorem 2.9, $h(\mathscr{A}(f))$ is compact if and only if

$$
\mathscr{P}(C(\Gamma) / \mathscr{A}(f))
$$

is compact. Since $C(\Gamma) / \mathscr{A}(f)$ is isomorphic with $C(\operatorname{spt} f)$, our problem is reduced to finding $f \in C(\Gamma)$ such that $f \notin P$ and spt $f$ satisfies the condition on $X$ in Corollary 5.5. In case $\iota(P)=\alpha \neq \omega_{1}$, simply choose $f \in C(\Gamma)$ so that $f(\alpha) \neq 0$ and $f$ vanishes on some neighborhood of $\omega_{1}$. In case $\iota(P)=\omega_{1}$, take $f=s$. As was noted earlier, if $g \in \mathscr{A}(s)$, then $g$ vanishes on a neighborhood of $\omega_{1}$. This implies that $\mathscr{A}(s) \subset O^{\omega_{1}} \subset P$. Hence, $s \notin P$ by Theorem 2.3. Furthermore, $\operatorname{spt} s=K$, which obviously satisfies the condition of Corollary 5.5.

If $\{h(f): f \in C(\Gamma)\}$ were a base for the topology of $\mathscr{P}(C(\Gamma))$, then for any $P$ such that $\iota(P)=\omega_{1}$ there would be an $f \in C(\Gamma)$ with

$$
P \in h(f) \subset h(\mathscr{A}(s)) .
$$

But $P \in h(f)$ implies $f\left(\omega_{1}\right)=0$, while $h(f) \subset h(\mathscr{A}(s))$ implies

$$
\operatorname{int}[Z(f) \cap Z(s)]=\operatorname{int}[Z(f) \cap L]=\emptyset .
$$

This is impossible, for as we observed earlier, any zero-set that contains $\omega_{1}$ also contains an interval of $L$.

Finally, let $S=\mathscr{A}(s)$ and

$$
I=\mathscr{A}(S)=\{f \in C(\Gamma): f[L]=\{0\}\} \text {. }
$$

Then $C(\Gamma) / I$ is isomorphic with $C(L)$, which in turn is isomorphic with $C\left(\boldsymbol{W}^{*}\right)$. In this last ring, the maximal ideal $\boldsymbol{M}^{\omega_{1}}=\left\{f \in C\left(\boldsymbol{W}^{*}\right): f\left(\omega_{1}\right)=0\right\}$ is a minimal prime ideal because it is precisely $O^{\omega 1}$. Hence, if

$$
M=\left\{f \in C(\Gamma): f\left(\omega_{1}\right)=0\right\},
$$

then $M / I$ is the corresponding minimal prime ideal of $C(\Gamma) / I$. But $M$ is not 
minimal, for it is a maximal ideal in $C(\Gamma)$ but does not coincide with the ideal $\left[f \in C(\Gamma): \omega_{1} \in \operatorname{int} Z(f)\right\}$.

5.8. ExAmple. A compact space $X$ such that $\mathscr{P}(C(X))$ is compact, but there is a function in $C(X)$ whose support is not a zero-set.

With $\boldsymbol{N}^{*}=\boldsymbol{N} \cup\{\infty\}$, the one-point compactification of $\boldsymbol{N}$, let $X$ be the following closed subspace of the product space $\Gamma \times N^{*}$ :

$$
X=\Gamma \times N^{*} \sim\left\{(m, n): m \in \Gamma, m<\omega_{0}, n \in N\right\} .
$$

$X$ is homeomorphic with what is obtained by adjoining to $W^{*} \times N^{*}$ a discrete sequence of points that converges to $\left(\omega_{1}, \infty\right)$. The function $s \in C(X)$ defined as $s(m, \infty)=1 / m$ if $0<m<\omega_{0}$ and $s(x)=0$ otherwise has a support which is not a zero-set. For, the point $\left(\omega_{1}, \infty\right)$ belongs to the support of $s$, and any function in $C(X)$ that vanishes at $\left(\omega_{1}, \infty\right)$ must also vanish at $(\alpha, \infty)$ for sufficiently large $\alpha<\omega_{1}$. Thus, $X$ does not satisfy the hypothesis of Theorem 5.6. $\mathscr{P}(C(X))$ is compact, however, by virtue of Corollary 5.5. To prove this, we note first of all, that for each $n \in N$, the subspace $X_{n}$ consisting of all points $(\alpha, n)$ of $X$ is homeomorphic with $W^{*}$. Each space $X_{n}$ does satisfy the condition of Corollary 5.5, and so there exists a function $g_{n} \in C\left(X_{n}\right)$ with the property that in the space $X_{n}, Z\left(f \mid X_{n}\right) \cup Z\left(g_{n}\right)=X_{n}$ and int $\left[Z\left(f \mid X_{n}\right) \cap Z\left(g_{n}\right)\right]=\emptyset$. We may assume, further, that $\left|g_{n}(x)\right| \leqq 1 / n$ for all $x \in X_{n}$. Now define

$$
f^{\prime}(x)= \begin{cases}g_{n}(x) & \text { if } x \in X_{n}, \\ 0 & \text { if } x=(\alpha, \infty) \text { with } \omega_{0}<\alpha \leqq \omega_{1} \text { or } \\ & \text { if } x \text { is in the support of } f, \\ 1 / m & \text { if } x=(m, \infty) \text { with } m<\omega_{0} \text { and } \\ & x \text { is not in the support of } f .\end{cases}
$$

It is easy to verify that $f^{\prime} \in C(X), Z(f) \cup Z\left(f^{\prime}\right)=X$, and $\operatorname{int}\left[Z(f) \cap Z\left(f^{\prime}\right)\right]$ $=\emptyset$.

5.9. ExAmple. A compact space $X$ such that no point of $\mathscr{P}(C(X))$ has a compact neighborhood.

The space $X=\beta N \sim N$ is known to be a compact, totally disconnected $F$-space that is not basically disconnected [2, pp.99, 100, and 210]. By Theorem $5.3(\mathrm{e}), \mathscr{P}(C(\beta N \sim N))$ is not compact. Now, the sets $h(\mathscr{A}(f))$, $f \in C(\beta \boldsymbol{N} \sim \boldsymbol{N}), f \neq 0$, constitute a base for the topology of $\mathscr{P}$. Since these sets are also closed, we can prove that no point of $\mathscr{P}$ has a compact neighborhood simply by showing that none of these sets is compact.

Since $\beta N \sim N$ is totally disconnected, the support of a given function $f \not \equiv 0$ contains a nonempty open-and-closed set $E$. If $e$ is the characteristic function of $E$, then $\mathscr{A}(f) \subset \mathscr{A}(e)$ so that $h(\mathscr{A}(f)) \supset h(\mathscr{A}(e))$. We shall now prove that the closed set $h(\mathscr{A}(e))$ is not compact. By Theorem 2.9, $h(\mathscr{A}(e))$ 
is homeomorphic with the space $\mathscr{P}(C(\beta N \sim N) / \mathscr{A}(e))$. Because $\mathscr{A}(e)$ is the set of all functions that vanish on $E$, the ring $C(\beta N \sim N) / \mathscr{A}(e)$ is isomorphic with $C(E)$. But $\beta N \sim N$ has the curious property that each of its nonempty open-and-closed subsets is homeomorphic with $\beta N \sim N$ [2, pp. 98-99]. Consequently, $h(\mathscr{A}(e))$, being homeomorphic with $\mathscr{P}(C(E))$, is not compact.

5.10. Example. If $X$ is any basically disconnected space that is not extremally disconnected $[2$, p.64], then $\mathscr{P}(C(X))$ has the same property because it is homeomorphic with $\beta X$ by Theorem 5.3 (d).

\section{REFERENCES}

1. L. Gillman, Rings with Hausdorff structure space, Fund. Math. 45 (1957), 1-16.

2. L. Gillman and M. Jerison, Rings of continuous functions, Van Nostrand, Princeton, N. J., 1960.

3. M. Henriksen and D. G. Johnson, On the structure of a class of lattice ordered algebras, Fund. Math. 50 (1961), 73-94.

4. I. Kaplansky, Topological representation of algebras. II, Trans. Amer. Math. Soc. 68 (1950), $62-75$.

5. J. Kist, Minimal prime ideals in commutative semigroups, Proc. London Math. Soc. (3) 13 (1963), 31-50.

6. C. W. Kohls, Prime ideals in rings of continuous functions. II, Duke Math. J. 25 (1958), 447-458.

7. N. H. McCoy, Rings and ideals, Carus Monograph No. 8, Math. Assoc. of America, Buffalo, N. Y., 1948.

Purdue University,

LAFA YETTE, INDIANA 